\title{
MORSE INEQUALITIES AND EMBEDDINGS FOR CR MANIFOLDS WITH CIRCLE ACTION
}

\author{
HENDRIK HERRMANN ${ }^{1, a}$ AND XIAOSHAN LI ${ }^{2, b}$ \\ ${ }^{1}$ Faculty of Mathematics and Natural Sciences, University of Wuppertal, Gaußstraße 20, \\ 42119 Wuppertal, Germany. \\ ${ }^{a}$ E-mail: post@hendrik-herrmann.de hherrmann@uni-wuppertal.de \\ ${ }^{2}$ School of Mathematics and Statistics, Wuhan University, Hubei 430072, China. \\ ${ }^{b}$ E-mail: xiaoshanli@whu.edu.cn
}

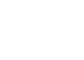

\begin{abstract}
In this paper, we would like to make a report on Morse inequalities and embeddings for CR manifolds with transversal CR circle action. The results are contained in 24], 23], 21], 20] and 25]. Furthermore, in the last section we will give an explicit example for $S^{1}$-equivariant Szegö kernels and their expansion on the sphere $S^{3}$ in $\mathbb{C}^{2}$ with respect to a family of transversal CR $S^{1}$-actions.
\end{abstract}

\section{Introduction}

Let $X$ be a real smooth manifold of dimension $2 n-1, n \geq 2$, together with a Lie group action of $S^{1}$. A CR structure (of codimension one or hypersurface type) on $X$ is a complex subbundle $T^{1,0} X$ of rank $n-1$ of the complexified tangent space $\mathbb{C} T X$ which is formally Frobenius integrable and satisfies $T^{1,0} X \cap \overline{T^{1,0} X}=\{0\}$. Given a Lie group action of $S^{1}$ we say that this $S^{1}$-action is $C R$ if it preserves $T^{1,0} X$ and transversal if its infinitesimal generator is transversal to the real part of $T^{1,0} X \oplus \overline{T^{1,0} X}$ at every point.

Received March 15, 2020 and in revised form June 15, 2020.

AMS Subject Classification: 32A25, 32V30.

Key words and phrases: Morse inequalities, Szegő kernel expansion, embeddings.

Hendrik Herrmann was partially supported by the Mathematical Institute, Academia Sinica, the School of Mathematics and Statistics, Wuhan University, and the Graduiertenkolleg 'Global Structures in Geometry and Analysis' at the University of Cologne.

Xiaoshan Li was supported by the National Natural Science Foundation of China (Grant No. 11871380). 
We start with a summary of results. In [24], we study the Fourier components of Kohn-Rossi cohomology on compact CR manifolds with transversal CR $S^{1}$-action. By using the Kohn's $L^{2}$-estimate on CR manifold, we show that without Levi-form assumptions the Fourier components of Kohn-Rossi cohomology are always finite dimensional. Then by studying the asymptotic properties of the Fourier components of the Szegő kernel function we get the asymptotic property of the dimension of the Fourier components of the Kohn-Rossi cohomology. A corollary of our results is that under the condition that the CR manifold is weakly pseudoconvex everywhere and strongly pseudoconvex at one point, the $\mathrm{CR}$ manifold admits many $S^{1}$-equivariant CR functions.

In [21], we study the equivariant embedding of a compact strongly pseudoconvex CR manifold with a transversal CR circle action. We develop an asymptotic expansion for the Fourier components of the Szegö kernels concerning CR functions which only lie in the positive Fourier components of the space of CR functions. Making use of the Szegö kernel asymptotic expansion, we can construct enough $S^{1}$-equivariant CR functions by which the $\mathrm{CR}$ manifold can be $\mathrm{CR}$ embedded into some $\mathbb{C}^{N}$. Moreover, in the last section of this paper, we give an example to describe explicitly such Szegö kernel asymptotic expansion with respect to the positive Fourier components of the space of $\mathrm{CR}$ functions on the unit sphere $S^{3}$ in $\mathbb{C}^{2}$.

It is well known that there are no compact Levi-flat CR manifolds in the complex Euclidean space. Thus, a natural question is whether the Levi-flat $\mathrm{CR}$ manifolds can $\mathrm{CR}$ embedded into the projective space by means of $\mathrm{CR}$ sections of a CR line bundle of positive curvature. This is the analogue of the Kodaira embedding theorem from complex geometry. In Kodaira's original proof of the Kodaira embedding theorem (see 34]), the Kodaira vanishing theorem is used to show that there are enough holomorphic sections with values in the large tensor powers of a holomorphic positive line bundle. In [23], we use the Szegö kernel asymptotic expansion method developed by Hsiao and Marinescu [19] to establish Morse inequalities on CR manifolds with a transversal CR circle action when such $\mathrm{CR}$ manifolds admit rigid CR line bundles. On such CR manifold, without any assumption on the positivity of the Levi-form we showed that a positive rigid CR line bundle is always big. 
In [25], we prove that a certain weighted Fourier-Szegö kernel admits a full asymptotic expansion and by using these asymptotics, we show that if $X$ admits a transversal CR locally free $S^{1}$-action and there is a rigid positive CR line bundle $L$ over $X$, then $X$ can be CR embedded into the projective space without any positivity assumptions on the Levi form. In particular, when $X$ is Levi-flat and admits a transversal CR $S^{1}$-action, we improve the regularity in the Kodaira embedding theorem of Ohsawa and Sibony [35] to $C^{\infty}$

\section{Morse Inequalities and Embeddings for CR Manifolds with Circle Action, I: without line bundle}

The problem of embedding CR manifolds attracts a lot of attention. A classical result of L. Boutet de Monvel [5] tells us that any compact strongly pseudoconvex CR manifold of real dimension greater or equal to five can be globally $\mathrm{CR}$ embedded into $\mathbb{C}^{N}$, for some $N \in \mathbb{N}$. The classical example of non-embeddable three dimensional strongly pseudoconvex CR manifold appears implicitly in the non-fillable example of pseudoconcave manifold by Grauert 16], Andreotti-Siu [2] and Rossi [36] and was explicited by Burns 7]. In [30] Lempert proved that a compact strongly pseudoconvex three dimensional CR manifold which admits a transversal CR circle action can always be CR embedded into the complex Euclidean space. Lempert proved that such CR manifold can always bound a strongly pseudoconvex complex surface. Then by Kohn's result [28] the Cauchy-Riemann operator $\bar{\partial}_{b}$ as an operator between $L^{2}$ spaces will have closed range. Thus by L. Boutet de Monvel [5] and Kohn [29] the CR manifold will be CR embedded into some complex Euclidean space. In [21], under the same condition as in 30], we showed that the functions in the CR embedding map can only come from the Fourier components of the space of CR functions. For further information on the embedding problem for CR manifolds we refer the reader to [12, 14, 15] and the references therein. Now we describe the results in [21].

Let $\left(X, T^{1,0} X\right)$ be a compact connected CR manifold of dimension $2 n-$ $1, n \geq 2$, where $T^{1,0} X$ is the given CR structure on $X$. That is, $T^{1,0} X$ is a subbundle of the complexified tangent bundle $\mathbb{C} T X$ of rank $n-1$, satisfying $T^{1,0} X \cap T^{0,1} X=\{0\}$, where $T^{0,1} X=\overline{T^{1,0} X}$, and $[\mathcal{V}, \mathcal{V}] \subset \mathcal{V}$, where $\mathcal{V}=C^{\infty}\left(X, T^{1,0} X\right)$. 
We assume that $X$ admits a $S^{1}$-action: $S^{1} \times X \rightarrow X,\left(e^{i \theta}, x\right) \mapsto e^{i \theta} \circ x$. Here, we use $e^{i \theta}(0 \leq \theta<2 \pi)$ to denote the $S^{1}$-action. Let $T \in C^{\infty}(X, T X)$ be the global real vector field induced by the $S^{1}$-action given as follows

$$
(T u)(x)=\left.\frac{\partial}{\partial \theta}\left(u\left(e^{i \theta} \circ x\right)\right)\right|_{\theta=0}, u \in C^{\infty}(X) .
$$

Definition 2.1. We say that the $S^{1}$-action is CR if

$$
\left[T, C^{\infty}\left(X, T^{1,0} X\right)\right] \subset C^{\infty}\left(X, T^{1,0} X\right),
$$

where [, ] is the Lie bracket between smooth vector fields on $X$. Furthermore, we say that the $S^{1}$-action is transversal if for each $x \in X$,

$$
\mathbb{C} T(x) \oplus T_{x}^{1,0}(X) \oplus T_{x}^{0,1} X=\mathbb{C} T_{x} X .
$$

For $x \in X$, we say that the period of $x$ is $\frac{2 \pi}{q}, q \in \mathbb{N}$, if $e^{i \theta} \circ x \neq x$ for every $0<\theta<\frac{2 \pi}{q}$ and $e^{i \frac{2 \pi}{q}} \circ x=x$. For each $q \in \mathbb{N}$, put

$$
X_{q}=\left\{x \in X ; \text { the period of } x \text { is } \frac{2 \pi}{q}\right\}
$$

and set $p=\min \left\{q \in \mathbb{N} ; X_{q} \neq \emptyset\right\}$. Without of loss of generality, we assume $p=1$. It is well-known that if $X$ is connected, then $X_{1}$ is an open and dense subset of $X$ (see [13] and also the appendix in [24]). We denote $X_{\text {reg }}:=X_{1}$. We call $x \in X_{\text {reg }}$ a regular point of the $S^{1}$-action. Let $X_{\text {sing }}$ be the complement of $X_{\text {reg }}$.

We assume throughout this paper that $\left(X, T^{1,0} X\right)$ is a compact connected CR manifold with a transversal CR locally free $S^{1}$-action and we denote by $T$ the global vector field induced by the $S^{1}$-action. Let $\omega_{0} \in$ $C^{\infty}\left(X, T^{*} X\right)$ be the global real 1-form determined by $\left\langle\omega_{0}, u\right\rangle=0$, for every $u \in T^{1,0} X \oplus T^{0,1} X$ and $\left\langle\omega_{0}, T\right\rangle=-1$.

Definition 2.2. For $x \in X$, the Levi-form $\mathcal{L}_{x}$ associated with the CR structure is the Hermitian quadratic form on $T_{x}^{1,0} X$ defined as follows. For any $U, V \in T_{x}^{1,0} X$, pick $\mathcal{U}, \mathcal{V} \in C^{\infty}\left(X, T^{1,0} X\right)$ such that $\mathcal{U}(x)=U, \mathcal{V}(x)=V$. Set

$$
\mathcal{L}_{x}(U, \bar{V})=\frac{1}{2 i}\left\langle[\mathcal{U}, \overline{\mathcal{V}}](x), \omega_{0}(x)\right\rangle
$$


where [, ] denotes the Lie bracket between smooth vector fields. Note that $\mathcal{L}_{x}$ does not depend on the choice of $\mathcal{U}$ and $\mathcal{V}$.

Definition 2.3. The CR structure on $X$ is called pseudoconvex at $x \in X$ if $\mathcal{L}_{x}$ is positive semidefinite. It is called strongly pseudoconvex at $x$ if $\mathcal{L}_{x}$ is positive definite. If the CR structure is (strongly) pseudoconvex at every point of $X$, then $X$ is called a (strongly) pseudoconvex CR manifold. In particular, we call $X$ a Levi-flat $\mathrm{CR}$ manifold if the Levi-form vanishes everywhere.

Let $\bar{\partial}_{b}: \Omega^{0, q}(X) \rightarrow \Omega^{0, q+1}(X)$ be the tangential Cauchy-Riemann operator where $\Omega^{0, q}(X)$ is the space of smooth $(0, q)$-forms on $X$ defined as in [8]. Set $\Omega_{m}^{0, q}(X)=\left\{u \in \Omega^{0, q}(X): L_{T} u=i m u\right\}$ where $L_{T}$ is the Lie derivative along direction $T$. From (2.2) and (2.3) one has $L_{T} \bar{\partial}_{b}=\bar{\partial}_{b} L_{T}$. Then it follows that $\bar{\partial}_{b}: \Omega_{m}^{0, q}(X) \rightarrow \Omega_{m}^{0, q+1}(X)$. In [24] we define

$$
H_{b, m}^{q}(X):=\frac{\operatorname{Ker} \bar{\partial}_{b}: \Omega_{m}^{0, q}(X) \rightarrow \Omega_{m}^{0, q+1}(X)}{\operatorname{Im} \bar{\partial}_{b}: \Omega_{m}^{0, q-1}(X) \rightarrow \Omega_{m}^{0, q}(X)}
$$

for $m \in \mathbb{Z}$. In particular, $H_{b, m}^{0}(X)=\left\{u \in C^{\infty}(X): T u=i m u, \bar{\partial}_{b} u=0\right\}$. We call $H_{b, m}^{0}(X)$ the $m$-th Fourier components of the space of CR functions.

\subsection{Morse inequalities}

From Kohn's $L^{2}$-estimate it is easy to see that $\operatorname{dim} H_{b, m}^{q}(X)$ is always finite dimensional without any assumption on the Levi-form. In [24], we studied the Morse inequalities for $\operatorname{dim} H_{b, m}^{q}(X)$ as $m \rightarrow \infty$. We have the following results.

Theorem 2.4. Let $X$ be a compact connected $C R$ manifold with a transversal $C R S^{1}$-action. Assume that $\operatorname{dim}_{\mathbb{R}} X=2 n-1, n \geq 2$. Then for every $q=0,1,2, \ldots, n-1$, we have

$$
\operatorname{dim} H_{b, m}^{q}(X) \leq \frac{m^{n-1}}{2 \pi^{n}} \int_{X(q)}\left|\operatorname{det} \mathcal{L}_{x}\right| d v_{X}(x)+o\left(m^{n-1}\right), m \rightarrow \infty .
$$

Here, $X(q)$ is a subset of $X$ where the Levi-form is nondegenerate and has exactly q-negative eigenvalues. $\operatorname{det} \mathcal{L}_{x}$ is the multiplication of the eigenvalues of the Levi-form with respect to a given $S^{1}$-invariant Hermitian metric on $X$. 
Theorem 2.5. Let $X$ be a compact connected $C R$ manifold with a transversal CR $S^{1}$-action. Assume that $\operatorname{dim}_{\mathbb{R}} X=2 n-1, n \geq 2$. For every $q=$ $0,1,2, \ldots, n-1$, as $m \rightarrow \infty$, we have

$\sum_{j=0}^{q}(-1)^{q-j} \operatorname{dim} H_{b, m}^{j}(X) \leq \frac{m^{n-1}}{2 \pi^{n}} \sum_{j=0}^{q}(-1)^{q-j} \int_{X(j)}\left|\operatorname{det} \mathcal{L}_{x}\right| d v_{X}(x)+o\left(m^{n-1}\right)$.

In particular, when $q=n-1$, as $m \rightarrow \infty$, we have the asymptotic RiemannRoch theorem

$$
\sum_{j=0}^{n-1}(-1)^{j} \operatorname{dim} H_{b, m}^{j}(X)=\frac{m^{n-1}}{2 \pi^{n}} \sum_{j=0}^{n-1}(-1)^{j} \int_{X(j)}\left|\operatorname{det} \mathcal{L}_{x}\right| d v_{X}(x)+o\left(m^{n-1}\right) .
$$

Recently, analogues to the Atiyah-Singer index theorem in complex geometry a CR index theorem is established in [9].

Corollary 2.6. Let $X$ be a compact connected $C R$ manifold with a transversal $C R S^{1}$-action. Assume that $\operatorname{dim}_{\mathbb{R}} X=2 n-1, n \geq 2$. If $X$ is weakly pseudoconvex and strongly pseudoconvex at a point, then as $m \rightarrow \infty$

$$
\operatorname{dim} H_{b, m}^{0}(X) \approx m^{n-1}, \operatorname{dim} H_{b, m}^{q}(X)=o\left(m^{n-1}\right) \text { for } q \geq 1
$$

In the main results above, we only consider the Morse inequalities for the positive Fourier component $\operatorname{dim} H_{b, m}^{q}(X)$ as $m \rightarrow \infty$. In fact, we also have the Morse inequalities for the negative Fourier component $H_{b, m}^{q}(X)$ as $m \rightarrow-\infty$. More precisely, we have

Theorem 2.7. Let $X$ be a compact connected $C R$ manifold with a transversal CR $S^{1}$-action. Assume that $\operatorname{dim}_{\mathbb{R}} X=2 n-1, n \geq 2$. For every $q=$ $0,1,2, \ldots, n-1$, as $m \rightarrow-\infty$, we have

$$
\begin{aligned}
& \operatorname{dim} H_{b, m}^{q}(X) \leq \frac{|m|^{n-1}}{2 \pi^{n}} \int_{X(n-1-q)}\left|\operatorname{det} \mathcal{L}_{x}\right| d v_{X}(x)+o\left(|m|^{n-1}\right), \\
& \sum_{j=0}^{q}(-1)^{q-j} \operatorname{dim} H_{b, m}^{j}(X) \\
& \quad \leq \frac{|m|^{n-1}}{2 \pi^{n}} \sum_{j=0}^{q}(-1)^{q-j} \int_{X(n-1-j)}\left|\operatorname{det} \mathcal{L}_{x}\right| d v_{X}(x)+o\left(|m|^{n-1}\right) .
\end{aligned}
$$


In particular, when $q=n-1$, as $m \rightarrow-\infty$, we have the asymptotic Riemann-Roch theorem

$$
\sum_{j=0}^{n-1}(-1)^{j} \operatorname{dim} H_{b, m}^{j}(X)=\frac{|m|^{n-1}}{2 \pi^{n}} \sum_{j=0}^{n-1}(-1)^{j} \int_{X(n-1-j)}\left|\operatorname{det} \mathcal{L}_{x}\right| d v_{X}(x)+o\left(|m|^{n-1}\right) .
$$

From Theorem 2.4, Theorem 2.5 and Theorem 2.7, we deduce

Theorem 2.8. Let $X$ be a compact connected $C R$ manifold of real dimension $2 n-1$ with a transversal $C R S^{1}$-action. Let $q \in\{0,1, \cdots, n-1\}$. Assume that the Levi form of $X$ has $q$ non-positive and $n-1-q$ non-negative eigenvalues everywhere. Then

$$
\begin{aligned}
& \operatorname{dim} H_{b, m}^{j}(X)=o\left(m^{n-1}\right), \text { as } m \rightarrow \infty, \text { for } j \neq q \\
& \operatorname{dim} H_{b, m}^{j}(X)=o\left(|m|^{n-1}\right), \text { as } m \rightarrow-\infty, \text { for } j \neq n-1-q .
\end{aligned}
$$

If moreover the Levi-form is non-degenerate at some point, then

$$
\begin{aligned}
& \operatorname{dim} H_{b, m}^{q}(X) \approx m^{n-1}, \text { as } m \rightarrow \infty \\
& \operatorname{dim} H_{b, m}^{n-1-q}(X) \approx|m|^{n-1}, \text { as } m \rightarrow-\infty \\
& \operatorname{dim} H_{b}^{q}(X)=\infty, \operatorname{dim} H_{b}^{n-1-q}(X)=\infty .
\end{aligned}
$$

In particularly, if $X$ is weakly pseudoconvex and strongly pseudoconvex at a point, then

$$
\operatorname{dim} H_{b, m}^{n-1}(X) \approx|m|^{n-1} \text { as } m \rightarrow-\infty
$$

and in particluar $\operatorname{dim} H_{b}^{n-1}(X)=\infty$. Moreover, $\operatorname{dim} H_{b, m}^{q}(X)=o\left(|m|^{n-1}\right)$ as $m \rightarrow-\infty$ for $q \leq n-2$.

Recently, when $X$ is only assumed to be weakly pseudoconvex the estimate $\operatorname{dim} H_{b, m}^{q}(X)=o\left(|m|^{n-1}\right)$ when $m \rightarrow-\infty$ is improved by Wang and Zhou [38, Theorem 1.3].

\subsection{Szegö kernel expansion and embeddings of CR manifolds}

In this section, we describe the results in [21]. From Corollary 2.6, we know that $\operatorname{dim} H_{b, m}^{0}(X) \approx m^{n-1}$ as $m \rightarrow \infty$ when $X$ is strongly pseudo- 
covnex. Hence, the space of CR functions which lie in the positive Fourier components of the space of CR functions is very large and thus a natural question is that whether $X$ can be CR embedded into some $\mathbb{C}^{N}$ by $\mathrm{CR}$ functions which lie in the positive Fourier components. In [21] we give an affirmative answer to this question. More precisely, we proved

Theorem 2.9. Let $X$ be a compact connected strongly pseudoconvex $C R$ manifold with a locally free transversal $C R S^{1}$-action. Then $X$ can be $C R$ embedded into some complex Euclidean space by the CR functions which lie in the positive Fourier components of the space of CR functions.

The main machinery to establish Theorem 2.9 are Szegö kernel expansion methods. In 21], making use of the complex stationary phase formula due to Melin-Sjöstrand [33] we have the following asymptotic expansion of the Fourier components of the Szegö kernel.

Theorem 2.10. Let $X$ be a compact strongly pseudoconvex CR manifold with a transversal $C R$ circle action. For $x_{0} \in X_{\mathrm{reg}}$, let $(z, \theta, \varphi)$ be canonical coordinates centered at $x_{0}$ and defined on a canonical patch $D_{1}=\{(z, \theta)$ : $\left.|z|<\varepsilon_{1},|\theta|<\pi\right\}$. Set $D=\left\{(z, \theta) \in \mathbb{C}^{n-1} \times \mathbb{R}:|z|<\varepsilon,|\theta|<\frac{\pi}{2}\right\} \Subset D_{1}$. Then on $D \times D$, we have

$$
S_{m}(x, y) \equiv \frac{1}{2 \pi} e^{i m\left(x_{2 n-1}-y 2 n-1+\Phi(z, w)\right)} \hat{b}(z, w, m) \bmod O\left(m^{-\infty}\right),
$$

where

$$
\begin{aligned}
& \hat{b}(z, w, m) \sim \sum_{j=0}^{\infty} m^{n-1-j} \hat{b}_{j}(z, w) \text { in } S_{\text {loc }}^{n-1}(1, \tilde{D} \times \tilde{D}), \\
& \hat{b}_{j}(z, w) \in C^{\infty}(\tilde{D} \times \tilde{D}), \quad j=0,1,2, \cdots, \\
& \hat{b}_{0}(z, z)=\pi^{-(n-1)}\left|\operatorname{det} \mathcal{L}_{x}\right|, \quad x=(z, 0), \quad \forall z \in \tilde{D} .
\end{aligned}
$$

Here, we set $\tilde{D}=\left\{z \in \mathbb{C}^{n-1}:|z|<\varepsilon\right\}$. In particular, we have

$$
S_{m}(x, x) \equiv \frac{1}{2 \pi} \hat{b}(z, z, m) \bmod O\left(m^{-\infty}\right) .
$$

Theorem 2.11. Let $X$ be a compact strongly pseudoconvex CR manifold with a transversal $C R$ circle action. Assume $x_{0} \in X_{k}, k>1$. Let $D_{1}, D$ 
and $(z, \theta, \varphi)$ be as above with $\frac{\pi}{k}-\epsilon$ replaced by $\delta_{1}$. For $k \nmid m$ we have $S_{m}\left(x, x_{0}\right)=0$ for all $x \in D$. For $k \mid m$ we have

$$
S_{m}\left(x, x_{0}\right) \equiv \frac{k}{2 \pi} e^{i m\left(x_{2 n-1}+\Phi(z, 0)\right)} \hat{b}(z, 0, m) \bmod O\left(m^{-\infty}\right)
$$

on D. In particular, given $k \mid m$ and $x=x_{0}$, we have

and

$$
S_{m}\left(x_{0}, x_{0}\right)=\frac{k}{2 \pi} \hat{b}(0,0, m)+O\left(m^{-\infty}\right)
$$

$$
\hat{b}(0,0, m) \sim \hat{b}_{0}(0,0) m^{n-1}+\hat{b}_{1}(0,0) m^{n-2}+\cdots
$$

in the sense that for any $N \in \mathbb{N}_{0}$ there exists $C_{N}>0$ independent of $m$ such that

$$
\left|\hat{b}(0,0, m)-\sum_{j=0}^{N} \hat{b}_{j}(0,0) m^{n-1-j}\right| \leq C_{N} m^{n-2-N}
$$

holds for all $m \in \mathbb{N}$.

In [20], we give a complete description of the diagonal expansion for the $m$-th Fourier component of the Szegö kernel function as $m \rightarrow+\infty$. We also obtain explicit formulas for the first three coefficients of such an expansion.

\section{Morse Inequalities and Embeddings for CR Manifolds with Circle Action, II: with line bundle}

The well-known Grauert-Riemenschneider conjecture states that a semipositive holomorphic line bundle on a compact complex manifold is big if it is positive at least at one point. This conjecture was first solved by Siu [37] by using the $\bar{\partial}$-equation method. Motivated by Siu's solution of GrauertRiemenschneider conjecture, Demailly [10] gave another proof of this conjecture by establishing the holomorphic Morse inequalities. The original proof of the holomorphic Morse inequalities was based partly on Siu's techniques and partly on an extension of Witten's analytic proof for the standard Morse inequalities. Natural problems [11] are to extend the Morse inequalities to cohomology associated to other operators than the $\bar{\partial}$-operator and to non-compact complex manifolds. Getzler [17] extended the Morse inequalities for $H_{b}^{q}\left(X, L^{k}\right)$ on a compact strongly pseudoconvex CR manifold $X$ for 
$1 \leq q \leq n-2$ where $L$ is a CR line bundle over $X$. Recently, Hsiao-Marinescu

[19] established the Morse inequalities for $H_{b}^{q}\left(X, L^{k}\right)$ on a general CR manifold which satisfies $Y(q)$ condition. Both Getzler's and Hsiao-Marinescu's work depend on the sign of the Levi-form on CR manifolds satisfying some algebraic conditions. So their work can not be used on Levi-flat CR manifolds which play an important part in CR geometry. In [23] we studied the Morse inequalities on CR manifolds which admit rigid CR line bundles. For Morse inequalities on non-compact complex manifolds which will be not focused on in this paper we refer the reader to [4, 26, 31, 32] and the references therein.

In our work [23] we do not need any restrictions on the behavior of the Levi-form of the CR manifold. From the Morse inequalities we have established, we prove that a rigid $\mathrm{CR}$ line bundle is big if it is positive. In order to describe this work, we first recall the definition of rigid CR line bundles.

\subsection{Rigid CR vector bundle}

In this subsection we use the notations from [22].

Definition 3.1. Let $D \subset X$ be an open set. We say that a function $u \in$ $C^{\infty}(D)$ is rigid if $T u=0$. We say that a function $u \in C^{\infty}(X)$ is CauchyRiemann (CR for short) if $\bar{\partial}_{b} u=0$. We say that $u \in C^{\infty}(X)$ is rigid CR if $\bar{\partial}_{b} u=0$ and $T u=0$.

The following definitions for CR vector bundles can be found in [27].

Definition 3.2. A complex vector bundle $(E, \pi, X)$ over $X$ is called $\mathrm{CR}$ vector bundle if

(i) $E$ is a CR manifold,

(ii) $\pi: E \rightarrow X$ is a $\mathrm{CR}$ submersion,

(iii) $E \oplus E \ni\left(\xi_{1}, \xi_{2}\right) \rightarrow \xi_{1}+\xi_{2} \in E$ and $\mathbb{C} \times E \ni(\lambda, \xi) \rightarrow \lambda \xi \in E$ are $\mathrm{CR}$ maps.

A smooth section $s \in \Gamma(U, E)$ defined on an open set $U \subset X$ is called $\mathrm{CR}$ section if the map $s: U \rightarrow E$ is $\mathrm{CR}$. 
Let $\left(E_{1}, \pi_{1}, X\right)$ and $\left(E_{2}, \pi_{2}, X\right)$ be two $\mathrm{CR}$ vector bundles over X. A map $F: E_{1} \rightarrow E_{2}$ is called a $\mathrm{CR}$ bundle isomorphism if $F$ is a $C^{\infty}$-diffeomorphism such that $F, F^{-1}$ are CR maps, $\pi_{2} \circ F=\pi_{1}$ and $F$ is fiberwise linear.

Given a $\mathrm{CR}$ vector bundle $(E, \pi, X)$ we find (see [27]) the linear partial differential operator $\bar{\partial}_{b}^{E}: \Gamma(X, E) \rightarrow \Gamma\left(X, E \otimes T^{* 0,1} X\right)$ satisfying

(a) $\bar{\partial}_{b}^{E}(f \cdot s)=s \bar{\partial}_{b}(f)+f \bar{\partial}_{b}^{E}(s)$ for all $f \in C^{\infty}(X)$ and $s \in \Gamma(X, E)$,

(b) $s \in \Gamma(U, E)$ is a CR section if and only if $\bar{\partial}_{b}^{E} s=0$.

Definition 3.3. A CR vector bundle $(E, \pi, X)$ of rank $r$ is called locally CR trivializable if for any point $p \in X$ there exists an open neighborhood $U \subset X$ such that $\left.E\right|_{U}$ is $\mathrm{CR}$ vector bundle isomorphic to the trivial $\mathrm{CR}$ vector bundle $U \times \mathbb{C}^{r}$.

The following lemma is well-known.

Lemma 3.4. Let $(E, \pi, X)$ be a $C R$ vector bundle. The following are equivalent:

(i) $(E, \pi, X)$ is locally $C R$ trivializable,

(ii) For any $p \in X$ there exists a smooth frame $\left\{f_{1}, \ldots, f_{r}\right\}$ of $\left.E\right|_{U}$ on an open neighborhood $U \subset X$ around $p$ such that $f_{1}, \ldots, f_{r}: U \rightarrow E$ are CR sections.

Remark 3.5. Let $\left\{f_{1}, \ldots, f_{r}\right\}$ be a frame of $\left.E\right|_{U}$ for some open set $U \subset X$. Then $\left\{f_{1}, \ldots, f_{r}\right\}$ is called CR frame if any $f_{k}, 1 \leq k \leq r$, is a CR section. Given two CR frames of $\left.E\right|_{U}$ we find by (a) and (b) that the corresponding transition matrix is $\mathrm{CR}$ in the sense that any entry is a CR function.

Definition 3.6. Let $\left(X, T^{1,0} X\right)$ be a $\mathrm{CR}$ manifold and let $T \in \Gamma(X, T X)$ be a $\mathrm{CR}$ vector field. A CR bundle lift of $T$ to $(E, \pi, X)$ is a linear partial differential operator $T^{E}: \Gamma(X, E) \rightarrow \Gamma(X, E)$ (with smooth coefficients) such that

(i) $T^{E}(f \cdot s)=T(f) \cdot s+f T^{E}(s)$ for all $f \in C^{\infty}(X)$ and $s \in \Gamma(X, E)$,

(ii) $\left[T^{E}, \bar{\partial}_{b}^{E}\right]=0$. 
In order to define $\left[T^{E}, \bar{\partial}_{b}^{E}\right]$ we need to define $T^{E}$ on $(0,1)$ forms with values in $E$ first. But this definition follows immediately from the fact that any $w \in \Gamma\left(X, E \otimes T^{* 0,1} X\right)$ locally can be written $w=\sum_{j=1}^{r} f_{j} \otimes \omega^{j}$ where $\left\{\omega^{j}\right\}$ are $(0,1)$-forms and $\left\{f_{j}\right\}$ are local frames of $E$ and that $T$ is defined also for $(0, q)$-forms using the Lie derivative.

Definition 3.7. Let $\left(X, T^{1,0} X\right)$ be a CR manifold and let $T \in \Gamma(X, T X)$ be a $\mathrm{CR}$ vector field. A $\mathrm{CR}$ vector bundle $(E, \pi, X)$ of rank $r$ over $X$ with a CR bundle lift $T^{E}$ of $T$ is called rigid CR (with respect to $T^{E}$ ) if for every point $p \in X$ there exists an open neighborhood $U$ around $p$ and a CR frame $\left\{f_{1}, \ldots, f_{r}\right\}$ of $\left.E\right|_{U}$ with $T^{E}\left(f_{j}\right)=0$ for $1 \leq j \leq r$.

A section $s \in \Gamma(X, E)$ is called a rigid $\mathrm{CR}$ section if $T^{E} s=0$ and $\bar{\partial}_{b}^{E} s=0$. The frame $\left\{f_{j}\right\}_{j=1}^{r}$ in Definition 3.7 is called a rigid CR frame of $\left.E\right|_{U}$. Note that it follows from Lemma 3.4 that any rigid CR vector bundle is locally CR trivializable.

Remark 3.8. In [24] and [25] we gave a definition of rigid CR vector bundle by assuming that the transition functions of a chosen local trivializing frame are rigid and CR. This definition is actually equivalent to the one given in Definition 3.7( see Lemma 3.9).

Lemma 3.9. Let $(E, \pi, X)$ be $C R$ vector bundle over a $C R$ manifold $\left(X, T^{1,0} X\right)$ of codimension $d$ and let $T \in \Gamma(X, T X)$ be a $C R$ vector field. The following are equivalent:

(i) $T$ has a $C R$ bundle lift $T^{E}$ such that $(E, \pi, X)$ is rigid $C R$ with respect to $T^{E}$.

(ii) There exist an open cover $\left\{U_{j}\right\}_{j \in \mathbb{N}}$ of $X$ and $C R$ frames $\left\{f_{1}^{j}, \ldots, f_{r}^{j}\right\}$ for $\left.E\right|_{U_{j}}, j \in \mathbb{N}$, such that the corresponding transition matrices are rigid $C R$ in the sense that any entry is a rigid $C R$ function.

Lemma 3.10. Let $\left(X, T^{1,0} X\right)$ be a $C R$ manifold with a transversal $C R S^{1}$ action. Let $T$ be the infinitesimal generator of the $S^{1}$-action. Let $(E, \pi, X)$ be a locally $C R$ trivializable $C R$ vector bundle of rank $r=1$. Assume that $T^{E}$ is a CR bundle lift of $T$ to $(E, \pi, X)$. Then $(E, \pi, X)$ is rigid $C R$. More precisely, for any $p \in X$ there exist an open neighborhood $U \subset X$ around $p$ and a $C R$ frame $\{f\}$ of $\left.E\right|_{U}$ with $T^{E}(f)=0$. 
Definition 3.11. Let $E$ be a rigid vector bundle over $X$. Let $\langle\cdot \mid \cdot\rangle_{E}$ be a Hermitian metric on $E$. We say that $\langle\cdot \mid \cdot\rangle_{E}$ is a rigid Hermitian metric if for every local rigid frame $f_{1}, \ldots, f_{r}$ of $E$, we have $T\left\langle f_{j} \mid f_{k}\right\rangle_{E}=0$, for every $j, k=1,2, \ldots, r$.

In order to simplify the notation we will denote by $\bar{\partial}_{b}, T$ the operators $\bar{\partial}_{b}^{E}, T^{E}$ where $E$ is any rigid $\mathrm{CR}$ vector bundle on $X$. Consider a locally CR trivializable CR line bundle $L$ over $X$ with a CR bundle lift of $T$. By Lemma 3.10 we find that $L$ is rigid $\mathrm{CR}$ with respect to that bundle lift. Hence there exists an open covering $\left(U_{j}\right)_{j=1}^{N}$ and a family of rigid CR trivializing frames $\left\{s_{j}\right\}_{j=1}^{N}$ with each $s_{j}$ defined on $U_{j}$ and the transition functions between different rigid CR frames are rigid CR functions. Let $L^{k}$ be the $k$-th tensor power of $L$. Then $\left\{s_{j}^{k}\right\}_{j=1}^{N}$ is a family of rigid CR trivializing frames on each $U_{j}$. Let $\bar{\partial}_{b}^{L^{k}}: \Omega^{0, q}\left(X, L^{k}\right) \rightarrow \Omega^{0, q+1}\left(X, L^{k}\right)$ be the tangential CauchyRiemann operator. Since $L^{k}$ is rigid $\mathrm{CR}$ we have $\bar{\partial}_{b} f=\bar{\partial}_{b} f_{j} \otimes s_{j}^{k}, T f=$ $\left(T f_{j}\right) \otimes s_{j}^{k}$ for any $f=f_{j} \otimes s_{j}^{k} \in \Omega^{0, q}\left(X, L^{k}\right)$ and

$$
T \bar{\partial}_{b}=\bar{\partial}_{b} T \text { on } \Omega^{0, q}\left(X, L^{k}\right) .
$$

Let $h^{L}$ be a Hermitian fiber metric on $L$. The local weight of $h^{L}$ with respect to a local rigid $\mathrm{CR}$ trivializing section $s$ of $L^{L}$ over an open subset $D \subset X$ is the function $\Phi \in C^{\infty}(D, \mathbb{R})$ for which

$$
|s(x)|_{h^{L}}^{2}=e^{-2 \Phi(x)}, x \in D .
$$

We denote by $\Phi_{j}$ the weight of $h^{L}$ with respect to $s_{j}$.

Definition 3.12. Let $L$ be a rigid CR line bundle and let $h^{L}$ be a Hermitian metric on $L$. The curvature of $\left(L, h^{L}\right)$ is the the Hermitian quadratic form $R^{L}=R^{\left(L, h^{L}\right)}$ on $T^{1,0} X$ defined by

$$
R_{p}^{L}(U, V)=\left\langle d\left(\bar{\partial}_{b} \Phi_{j}-\partial_{b} \Phi_{j}\right)(p), U \wedge \bar{V}\right\rangle, \quad U, V \in T_{p}^{1,0} X, p \in U_{j}
$$

Due to [19, Proposition 4.2], $R^{L}$ is a well-defined global Hermitian form, since the transition functions between different frames $s_{j}$ are annihilated by $T$.

Definition 3.13. We say that $\left(L, h^{L}\right)$ is positive if the associated curvature $R_{x}^{L}$ is positive definite at every $x \in X$. 


\subsection{Morse inequalities for $\mathrm{CR}$ manifolds with circle action}

Let $\left(X, T^{1,0} X\right)$ be a compact connected CR manifold of dimension $2 n-1$, $n \geqslant 2$. Let $L$ be a rigid CR line bundle over $X$. For every $u \in \Omega^{0, q}\left(X, L^{k}\right)$, we can define $T u \in \Omega^{0, q}\left(X, L^{k}\right)$ in a natural way since locally we can find rigid $\mathrm{CR}$ frames such that the transition functions are rigid and CR. Moreover, we have

$$
T \bar{\partial}_{b}=\bar{\partial}_{b} T \text { on } \Omega^{0, q}\left(X, L^{k}\right),
$$

where $\bar{\partial}_{b}: \Omega^{0, q}\left(X, L^{k}\right) \rightarrow \Omega^{0, q+1}\left(X, L^{k}\right)$ denotes the tangential CauchyRiemann operator. For every $m \in \mathbb{Z}$, the $m$-th Fourier component of $\bar{\partial}_{b}$ cohomology is given by

$$
H_{b, m}^{q}\left(X, L^{k}\right):=\frac{\operatorname{Ker} \bar{\partial}_{b}: \Omega_{m}^{0, q}\left(X, L^{k}\right) \rightarrow \Omega_{m}^{0, q+1}\left(X, L^{k}\right)}{\operatorname{Im} \bar{\partial}_{b}: \Omega_{m}^{0, q-1}\left(X, L^{k}\right) \rightarrow \Omega_{m}^{0, q}\left(X, L^{k}\right)}
$$

Without any Levi curvature assumption, for any $m \in \mathbb{Z}$ and any $q=$ $0,1,2 \ldots, n-1$, we have

$$
\operatorname{dim} H_{b, m}^{q}\left(X, L^{k}\right)<\infty
$$

Fix $\lambda \geq 0$ and set $H_{b, \leq \lambda}^{q}\left(X, L^{k}\right):=\bigoplus_{m \in \mathbb{Z},|m| \leq \lambda} H_{b, m}^{q}\left(X, L^{k}\right)$. In this work, we study the asymptotic behavior of the space $H_{b, \leq k \delta}^{q}\left(X, L^{k}\right)$ and its partial Szegő kernel. Our main results are the following

Theorem 3.14. For $k$ large and for every $q=0,1,2, \ldots, n-1$, we have $\operatorname{dim} H_{b, \leq k \delta}^{q}\left(X, L^{k}\right) \leq(2 \pi)^{-n} \frac{(-1)^{q}}{(n-1) !} k^{n} \int_{X} \int_{\mathbb{R}_{x, q} \cap[-\delta, \delta]}\left(i \mathcal{R}_{x}^{L}+2 s i \mathcal{L}_{x}\right)^{n-1} \wedge\left(-\omega_{0}\right)+o\left(k^{n}\right)$,

where $\mathcal{R}_{x}^{L}$ denotes the curvature of $L, \mathcal{L}_{x}$ denotes the Levi form of $X, \omega_{0}$ is the unique global non-vanishing real one form determined by $\left\langle\omega_{0}, u\right\rangle=0$, $\forall u \in T^{1,0} X \oplus T^{0,1} X$ and $\left\langle\omega_{0}, T\right\rangle=-1$ and

$$
\begin{array}{r}
\mathbb{R}_{x, q}:=\left\{s \in \mathbb{R}: \mathcal{R}_{x}^{L}+2 s \mathcal{L}_{x} \text { has exactly } q\right. \text { negative and } \\
n-1-q \text { positive eigenvalues }\} .
\end{array}
$$

Note that $\mathcal{R}_{x}^{L}, \mathcal{L}_{x} \in T_{x}^{* 1,0} X \wedge T_{x}^{* 0,1} X$ (see Definition 2.2). Hence, $\left(\mathcal{R}_{x}^{L}+\right.$ $\left.2 s \mathcal{L}_{x}\right)^{n-1} \wedge\left(-\omega_{0}(x)\right)$ is a global $2 n-1$ form on $X$. Any Hermitian fiber 
metric $h^{L}$ on $L$ induces a curvature $\mathcal{R}^{L}$. It is easy to see that the integral in (3.7) does not depend on the choice of Hermitian fiber metric of $L$.

Theorem 3.15. For $k$ large and for every $q=0,1,2, \ldots, n-2$, we have

$$
\begin{aligned}
& \sum_{j=0}^{q}(-1)^{q-j} \operatorname{dim} H_{b, \leq k \delta}^{j}\left(X, L^{k}\right) \\
& \leq(2 \pi)^{-n} \frac{k^{n}}{(n-1) !}(-1)^{q} \sum_{j=0}^{q} \int_{X} \int_{\mathbb{R}_{x, j} \cap[-\delta, \delta]}\left(i \mathcal{R}_{x}^{L}+i 2 s \mathcal{L}_{x}\right)^{n-1} \wedge\left(-\omega_{0}(x)\right) d s+o\left(k^{n}\right)
\end{aligned}
$$

and when $q=n-1$, we have asymptotic Riemann-Roch-Hirzebruch theorem

$$
\begin{aligned}
& \sum_{j=0}^{n-1}(-1)^{j} \operatorname{dim} H_{b, \leq k \delta}^{j}\left(X, L^{k}\right) \\
& =(2 \pi)^{-n} \frac{k^{n}}{(n-1) !} \sum_{j=0}^{n-1} \int_{X} \int_{\mathbb{R}_{x, j} \cap[-\delta, \delta]}\left(i \mathcal{R}_{x}^{L}+i 2 s \mathcal{L}_{x}\right)^{n-1} \wedge\left(-\omega_{0}(x)\right) d s+o\left(k^{n}\right)
\end{aligned}
$$

Assume that $\mathcal{R}^{L}$ is positive. If $\delta>0$ is small enough then $\mathbb{R}_{x, j} \cap[\delta, \delta]=\emptyset$, $\forall x \in X$ and for every $j=1,2, \ldots, n-1$. From this observation, (3.7) and (3.10), we conclude that

$$
\begin{aligned}
& \operatorname{dim} H_{b, \leq k \delta}^{0}\left(X, L^{k}\right) \\
& =(2 \pi)^{-n} \frac{1}{(n-1) !} k^{n} \int_{X} \int_{\mathbb{R}_{x, 0} \cap[-\delta, \delta]}\left(i \mathcal{R}_{x}^{L}+i 2 s \mathcal{L}_{x}\right)^{n-1} \wedge\left(-\omega_{0}(x)\right) d s+o\left(k^{n}\right) .
\end{aligned}
$$

Hence, $\operatorname{dim} H_{b, \leq k \delta}^{0}\left(X, L^{k}\right) \approx k^{n}$. We conclude that

Theorem 3.16. If $L$ is a positive rigid $C R$ line bundle, then $L$ is big, that is, $\operatorname{dim} H_{b}^{0}\left(X, L^{k}\right) \gtrsim k^{n}$ when $k \gg 1$.

\subsection{Kodaira embedding theorems for CR manifolds with circle action}

Ohsawa and Sibony [35] constructed for every $\kappa \in \mathbb{N}$ a CR projective embedding of class $C^{\kappa}$ of a Levi-flat CR manifold by using $\bar{\partial}$-estimates. A natural question is whether we can improve the regularity to $\kappa=\infty$. Adachi [1] showed that the answer is no, in general. The analytic difficulty of 
this problem comes from the fact that the Kohn Laplacian is not hypoelliptic on Levi flat manifolds. In [25] we give an affirmative answer to this question under the condition that the CR manifold admits a transversal CR $S^{1}$-action and a rigid positive $\mathrm{CR}$ line bundle. Now we describe the results in [25].

For every $m \in \mathbb{Z}$, let $L_{m}^{2}\left(X, L^{k}\right) \subset L^{2}\left(X, L^{k}\right)$ be the completion of $C_{m}^{\infty}\left(X, L^{k}\right)$ with respect to the Hermitian inner product $(\cdot \mid \cdot)$ induced by the $S^{1}$-invariant Hermitian metric on $X$ and the rigid Hermitian fiber metric $h^{L}$ for $L$. Let

$$
Q_{m, k}^{(0)}: L^{2}\left(X, L^{k}\right) \rightarrow L_{m}^{2}\left(X, L^{k}\right)
$$

be the orthogonal projection with respect to $(\cdot \mid \cdot)$. We recall the weighted Fourier Szegö projection defined in [25]. Fix $\delta>0$ and a function

$$
\tau_{\delta} \in C_{0}^{\infty}((-\delta, \delta)), \quad 0 \leq \tau_{\delta} \leq 1, \tau_{\delta}=1 \text { on }\left[-\frac{\delta}{2}, \frac{\delta}{2}\right] .
$$

Let $F_{k, \delta}: L^{2}\left(X, L^{k}\right) \rightarrow L^{2}\left(X, L^{k}\right)$ be the bounded operator given by $F_{k, \delta}(u)$ $=\sum_{m \in \mathbb{Z}} \tau_{\delta}\left(\frac{m}{k}\right) Q_{m, k}^{(0)}(u), \forall u \in L^{2}\left(X, L^{k}\right)$. For every $\lambda>0$, we consider the partial Szegö projector $\Pi_{k, \leq \lambda}: L^{2}\left(X, L^{k}\right) \rightarrow H_{b, \leq \lambda}^{0}\left(X, L^{k}\right)$ which is the orthogonal projection on the space of equivariant CR functions of degree less than $\lambda$. The weighted Fourier-Szegö operator is defined as follows.

$$
P_{k, \delta}:=F_{k, \delta} \circ \Pi_{k, \leq k \delta} \circ F_{k, \delta}: L^{2}\left(X, L^{k}\right) \rightarrow H_{b, \leq k \delta}^{0}\left(X, L^{k}\right) .
$$

The Schwartz kernel of $P_{k, \delta}$ with respect to $d v_{X}$ is the smooth function $P_{k, \delta}(x, y) \in L_{x}^{k} \otimes\left(L_{y}^{k}\right)^{*}$ satisfying

$$
\left(P_{k, \delta} u\right)(x)=\int_{X} P_{k, \delta}(x, y) u(y) d v_{X}(y), u \in L^{2}\left(X, L^{k}\right) .
$$

Let $P_{k, \delta, s}: L_{\text {comp }}^{2}(D) \rightarrow L^{2}(D)$ be the localization of $P_{k, \delta}$ with respect to a local rigid frame $s$ of $L$ over an open subset $D \subset X$. Here, $L_{\text {comp }}^{2}(D)$ is a subspace of $L^{2}(D)$ with elements having compact support in $D$. Let $P_{k, \delta, s}(x, y) \in C^{\infty}(D \times D)$ be the Schwartz kernel of $P_{k, \delta, s}$ with respect to $d v_{X}$, defined as in (3.14). Then we can describe the structure of the localized Fourier-Szegö kernel $P_{k, \delta, s}(x, y)$.

Theorem 3.17. Let $X$ be a compact $C R$ manifold with a transversal $C R$ locally free $S^{1}$-action and let $L$ be a positive rigid $C R$ line bundle on $X$. 
Consider a point $p \in X$ and a canonical coordinate neighborhood $(D, x=$ $\left.\left(x_{1}, \ldots, x_{2 n-1}\right)\right)$ centered at $p=0$. Let $s$ be a local rigid $C R$ frame of $L$ on $D$ and set $|s|_{h}^{2}=e^{-2 \Phi}$. Fix $\delta>0$ small enough and $D_{0} \Subset D$. Then

$$
P_{k, \delta, s}(x, y)=\int_{\mathbb{R}} e^{i k \varphi(x, y, t)} g(x, y, t, k) d t+O\left(k^{-\infty}\right) \text { on } D_{0} \times D_{0},
$$

where $\varphi \in C^{\infty}(D \times D \times(-\delta, \delta))$ is a phase function such that for some constant $c>0$ we have

$$
\begin{aligned}
& \left.d_{x} \varphi(x, y, t)\right|_{x=y}=-2 \operatorname{Im} \bar{\partial}_{b} \Phi(x)-t \omega_{0}(x), \\
& \left.d_{y} \varphi(x, y, t)\right|_{x=y}=2 \operatorname{Im} \bar{\partial}_{b} \Phi(x)+t \omega_{0}(x), \operatorname{Im} \varphi(x, y, t) \geq c|z-w|^{2}, \\
& (x, y, t) \in D \times D \times(-\delta, \delta), x=\left(z, x_{2 n-1}\right), y=\left(w, y_{2 n-1}\right), \\
& \operatorname{Im} \varphi(x, y, t)+\left|\frac{\partial \varphi}{\partial t}(x, y, t)\right|^{2} \geq c|x-y|^{2},(x, y, t) \in D \times D \times(-\delta, \delta), \\
& \varphi(x, y, t)=0 \text { and } \frac{\partial \varphi}{\partial t}(x, y, t)=0 \text { if and only if } x=y,
\end{aligned}
$$

and $g(x, y, t, k) \in S_{\text {loc }}^{n}(1 ; D \times D \times(-\delta, \delta)) \cap C_{0}^{\infty}(D \times D \times(-\delta, \delta))$ is a symbol with expansion

$$
g(x, y, t, k) \sim \sum_{j=0}^{\infty} g_{j}(x, y, t) k^{n-j} \text { in } S_{\mathrm{loc}}^{n}(1 ; D \times D \times(-\delta, \delta)),
$$

and for $x \in D_{0}$ and $|t|<\delta$ we have

$$
g_{0}(x, x, t)=(2 \pi)^{-n}\left|\operatorname{det}\left(R_{x}^{L}+2 t \mathcal{L}_{x}\right)\right|\left|\tau_{\delta}(t)\right|^{2} .
$$

Corollary 3.18. In the conditions of Theorem 3.17 we have as $k \rightarrow \infty$,

$$
P_{k, \delta}(x, x) \sim \sum_{j=0}^{\infty} k^{n-j} b_{j}(x) \text { in } S_{\mathrm{loc}}^{n}(1 ; X)
$$

where $b_{j}(x) \in C^{\infty}(X), j=0,1,2, \ldots$, and

$$
b_{0}(x)=(2 \pi)^{-n} \int_{\mathbb{R}}\left|\operatorname{det}\left(R_{x}^{L}+2 t \mathcal{L}_{x}\right)\right|\left|\tau_{\delta}(t)\right|^{2} d t,
$$

with $\tau_{\delta} \in C_{0}^{\infty}(\mathbb{R})$ introduced in (3.12).

In [25], we give an example to show that the Szegő kernel for $H_{b, \leq k \delta}^{0}(X$, 
$\left.L^{k}\right)$ does not admit an asymptotic expansion as $k \rightarrow \infty$. Thus, the weighted Szegő kernel is necessary for the existence of an expansion.

We define now the Kodaira map. Consider an open set $D \subset X$ which is $S^{1}$-invariant and let $s: D \rightarrow L$ be a local rigid $\mathrm{CR}$ trivializing section on $D$. For any $u \in C^{\infty}\left(X, L^{k}\right)$ we write $u(x)=s^{k}(x) \otimes \widetilde{u}(x)$ on $D$, with $\widetilde{u} \in C^{\infty}(D)$. Let $\left\{f_{j}\right\}_{j=1}^{d_{k}}$ be an orthonormal basis of $H_{b, \leq k \delta}^{0}\left(X, L^{k}\right)$ with respect to $(\cdot \mid \cdot)$ such that $f_{j} \in H_{b, m_{j}}^{0}\left(X, L^{k}\right)$ and set $g_{j}=F_{k, \delta} f_{j}, 1 \leq j \leq d_{k}$. The Kodaira map is defined on $D$ by

$$
\begin{aligned}
\Phi_{k, \delta}: D & \longrightarrow \mathbb{C P}^{d_{k}-1}, \\
x & \longmapsto\left[F_{k, \delta} f_{1}, \ldots, F_{k, \delta} f_{d_{k}}\right]:=\left[\widetilde{g}_{1}(x), \ldots, \widetilde{g}_{d_{k}}(x)\right], \text { for } x \in D .
\end{aligned}
$$

Theorem 3.19. Let $\left(X, T^{1,0} X\right)$ be a compact $C R$ manifold with a transversal CR locally free $S^{1}$-action. Assume there is a rigid positive $C R$ line bundle $L$ over $X$. Then there exists $\delta_{0}>0$ such that for all $\delta \in\left(0, \delta_{0}\right)$ there exists $k(\delta)$ so that for $k>k(\delta)$ and any orthonormal basis $\left\{f_{j}\right\}_{j=1}^{d_{k}}$ of $H_{b, \leq k \delta}^{0}\left(X, L^{k}\right)$ with respect to $(\cdot \mid \cdot)$ such that the map $\Phi_{k, \delta}$ introduced in (3.21) is a smooth CR embedding.

In [18], it was shown that if $X$ admits a transversal CR locally free $S^{1}$ action and there is a rigid positive CR line bundle $L$ over $X$, then $X$ can be $\mathrm{CR}$ embedded into projective space under the assumption that condition $Y(0)$ holds on $X$. In Theorem 3.19 we remove the Levi curvature assumption $Y(0)$ used in [18].

As a consequence of Theorem 3.19 we obtain an embedding result for Levi-flat CR manifolds.

Corollary 3.20. Let $X$ be a compact Levi-flat $C R$ manifold. Assume that $X$ admits a transversal $C R$ locally free $S^{1}$-action and a positive rigid $C R$ line bundle. Then there exists $\delta_{0}>0$ such that for all $\delta \in\left(0, \delta_{0}\right)$ there exists $k(\delta)$ so that for $k>k(\delta)$ the map $\Phi_{k, \delta}$ introduced in (3.21) is a $C^{\infty} C R$ embedding of $X$ in $\mathbb{C P}^{d_{k}-1}$ which is $S^{1}$-equivariant with respect to weighted diagonal actions. 


\section{Equivariant Szegő Kernels on $S^{3}$}

In this section we will give an explicit example for $S^{1}$-equivariant Szegő kernels and their expansion. We will study the 3 -sphere $S^{3}$ as the boundary of the open unit ball $B^{2}$ in $\mathbb{C}^{2}$ together with a family of $\mathrm{CR} S^{1}$-actions. On the one hand for each of this actions we have to construct a metric on $S^{3}$ satisfying several properties (see the definition of rigid Hermitian metric). We will do this in Section 4.1 and we will also calculate the determinant of the Levi form (see Lemma 4.7) there. On the other hand we will compute the Szegö kernel for positive Fourier coefficients in such settings explicit by constructing an orthonormal basis for the function spaces in question (see Section 4.2, Theorem 4.10). In Section 4.3 we will discuss the example in context of the general theory as presented in Section 2.2.

A point in $\mathbb{C}^{2}$ or $S^{3}$ is always denoted by $z=\left(z_{1}, z_{2}\right)$.

\subsection{Setting}

Let $X=S^{3}=\left\{\rho(z):=\left|z_{1}\right|^{2}+\left|z_{2}\right|^{2}=1\right\} \subset \mathbb{C}^{2}$ be the 3-sphere together with the CR structure given by $T^{1,0} X=\mathbb{C} T X \cap T^{1,0} \mathbb{C}^{2}=\mathbb{C} Z$ where

$$
Z_{z}=\gamma(z)^{-1}\left(\bar{z}_{2} \frac{\partial}{\partial z_{1}}-\bar{z}_{1} \frac{\partial}{\partial z_{2}}\right)
$$

for $z \in X$ and $\gamma$ is a smooth non vanishing function defined on $\mathbb{C}^{2}$. Moreover, let $\ell: X \rightarrow \mathbb{C}^{2}$ denote the inclusion map. For $n \in \mathbb{Z}$ consider the holomorphic $S^{1}$-action $\tilde{\mu}: S^{1} \times \mathbb{C}^{2} \rightarrow \mathbb{C}^{2},\left(e^{i \theta}, z\right) \mapsto\left(e^{i \theta} z_{1}, e^{i n \theta} z_{2}\right)$. Then $\tilde{\mu}$ restricts to a CR action on $X$ which we will denote by $\mu$. One has

$$
T_{z}:=\frac{\partial}{\partial \theta} \mu\left(e^{i \theta}, z\right)_{\left.\right|_{\theta=0}}=i\left(z_{1} \frac{\partial}{\partial z}{ }_{1}-\bar{z}_{1} \frac{\partial}{\partial \bar{z}_{1}}+n\left(z_{2} \frac{\partial}{\partial z_{2}}-\bar{z}_{2} \frac{\partial}{\partial \bar{z}_{2}}\right)\right)
$$

for $z \in X$ and $T$ (resp. $Z$ ) can be extended in an obvious way to a vector field on $\mathbb{C}^{2}$ also denoted by $T$ (resp. $Z$ ). We further assume that $\left|\gamma_{\mid X}\right|$ is $\mu$-invariant. The following theorem describes crucial properties for the CR $S^{1}$-action $\mu$ on $X$ for several $n$ (see Definition 2.1 for the definition of transversal CR $S^{1}$-action). 
Theorem 4.1. One has that $\mu$ is:

(i) locally free $\Leftrightarrow n \neq 0$

(ii) globally free $\Leftrightarrow n \in\{ \pm 1\}$

(iii) transversal $\Leftrightarrow n>0$

Proof. For $n \neq 0$ one has that $T_{z}=0$ implies $z=0 \notin X$. On the other hand $T_{(0,1)}=0$ when $n=0$ which proves (i). In order to prove (ii) one observe that for $z=\left(0, z_{2}\right) \in X, \mu\left(e^{i \theta}, z\right)=z$ if and only if $n \theta \in 2 \pi \mathbb{Z}$ and for $z=\left(z_{1}, z_{2}\right) \in X$ such that $z_{1} \neq 0$ one has $\mu\left(e^{i \theta}, z\right)=z$ if and only if $\theta \in 2 \pi \mathbb{Z}$. For the third part we define a 1 -form $\alpha$ on $\mathbb{C}^{2}$ by

$$
\alpha_{z}=\frac{i}{2}\left(z_{1} d \bar{z}_{1}-\bar{z}_{1} d z_{1}+z_{2} d \bar{z}_{2}-\bar{z}_{2} d z_{2}\right) .
$$

Then $\alpha \neq 0$ in a neighbourhood around $X$ and since $\left.\alpha(\operatorname{grad} \rho)\right|_{X}=0($ where $\rho$ is a defining function for $X)$ one has that $\ell^{*} \alpha$ defines a non vanishing 1form on $X$. One has $\alpha(Z)=\alpha(\bar{Z})=0$ and $\alpha(T)_{z}=\left|z_{1}\right|^{2}+n\left|z_{2}\right|^{2}$. Thus, for $n>0$ one obtains $\alpha(T)>0$ which implies $\mathbb{C} T \cap T^{1,0} X \oplus T^{0,1} X=0$. Given $n \leq 0$ set $z_{1}=\sqrt{-n /(1-n)}, z_{2}=\sqrt{1 /(1-n)}$ and $z=\left(z_{1}, z_{2}\right)$. Then $|z|^{2}=1$ and

$$
\alpha(T)_{z}=\frac{-n}{1-n}+\frac{n}{1-n}=0=\alpha(Z)_{z}=\alpha(\bar{Z})_{z} .
$$

Since $\ell^{*} \alpha_{z} \neq 0$ and the linear independency of $Z$ and $\bar{Z}$ one has $T_{z} \in$ $T_{z}^{1,0} X \oplus T_{z}^{0,1} X$.

Remark 4.2. For $|n|>1$ one can write $X=X_{\text {reg }} \dot{\cup} X_{n}$ where $X_{\text {reg }}=\{z \in$ $\left.X \mid z_{1} \neq 0\right\}$.

Given $m \in \mathbb{Z}, m \geq 0$, consider the space

$$
\mathbb{C}\left[z_{1}, z_{2}\right]_{m}:=\operatorname{span}_{\mathbb{C}}\left(\left\{z \mapsto z_{1}^{l} z_{2}^{k} \mid l, k \geq 0, m=l+n k\right\}\right) .
$$

Lemma 4.3. We have that the linear map $\ell^{*}: \mathbb{C}\left[z_{1}, z_{2}\right]_{m} \rightarrow H_{b, m}^{0}(X)$ is well defined and injective.

Proof. Since $k, l \geq 0$ one has $\left(z \mapsto z_{1}^{l} z_{2}^{k}\right)_{\left.\right|_{X}} \in H_{b}^{0}(X)$ as the restriction of a holomorphic function and $2 \pi z_{1}^{l} z_{2}^{k}=\int_{0}^{2 \pi}\left(e^{i \theta} z_{1}\right)^{l}\left(e^{i n \theta} z_{2}\right)^{k} e^{-i m \theta} d \theta$ for all $z \in$ $X$ if and only if $m=l+n k$ (see (2.6) for the definition of $H_{b, m}^{0}(X)$ ). Hence 
$\ell^{*} \mathbb{C}\left[z_{1}, z_{2}\right]_{m} \subset H_{b, m}^{0}(X)$. From the Bochner's extension theorem it follows that every smooth function in $H_{b}^{0}(X)$ extends uniquely to a function in $H^{0}\left(B^{2}\right) \cap C^{\infty}\left(\overline{B^{2}}\right)$ where $H^{0}\left(B^{2}\right)$ denotes the space of holomorphic functions on $B^{2}$. Hence for $f \in \mathbb{C}\left[z_{1}, z_{2}\right]_{m}$ with $\ell^{*} f=0$ we must have $f=0$ which proves the injectivity of $\ell^{*}$ restricted to $\mathbb{C}\left[z_{1}, z_{2}\right]_{m}$.

As a direct consequence of Lemma 4.3 we have

$$
\operatorname{dim}\left(\ell^{*} \mathbb{C}\left[z_{1}, z_{2}\right]_{m}\right)= \begin{cases}\left\lfloor\frac{m}{n}\right\rfloor+1, & \text { for } n \geq 1, \\ \infty, & \text { else }\end{cases}
$$

and hence $\operatorname{dim} H_{b, m}^{0}(X)=\infty$ in the case $n \leq 0$.

Remark 4.4. We observe the importance of having a transversal CR $S^{1}$ action for $H_{b, m}^{0}(X)$ being finite dimensional.

Lemma 4.5. Given $n \geq 1$ we have $\ell^{*} \mathbb{C}\left[z_{1}, z_{2}\right]_{m}=H_{b, m}^{0}(X)$.

Proof. By Lemma 4.3 we have $\ell^{*} \mathbb{C}\left[z_{1}, z_{2}\right]_{m} \subset H_{b, m}^{0}(X)$. On the other hand choose an arbitrary $f \in H_{b, m}^{0}(X)$. By the Bochner's extension theorem we find a uniquely determined $F \in H^{0}\left(B^{2}\right) \cap C^{\infty}\left(\overline{B^{2}}\right)$ such that $\left.F\right|_{X}=f$. Put

$$
\tilde{F}(z)=\frac{1}{2 \pi} \int_{0}^{2 \pi} F\left(\tilde{\mu}\left(e^{i \theta}, z\right)\right) e^{-i m \theta} d \theta \text { for all } z \in \overline{B^{2}} .
$$

Then $\tilde{F} \in H^{0}\left(B^{2}\right) \cap C^{\infty}\left(\overline{B^{2}}\right)$ and for any $z \in X$ we have

$$
\tilde{F}(z)=\frac{1}{2 \pi} \int_{0}^{2 \pi} f\left(\tilde{\mu}\left(e^{i \theta}, z\right)\right) e^{-i m \theta} d \theta=f(z)
$$

which implies $\left.\tilde{F}\right|_{X}=f$. By the uniqueness of the extension we find $F=\tilde{F}$ and hence

$$
F(z)=\frac{1}{2 \pi} \int_{0}^{2 \pi} F\left(\tilde{\mu}\left(e^{i \theta}, z\right)\right) e^{-i m \theta} d \theta \text { for all } z \in B^{2} .
$$

Since $F$ is holomorphic on $B^{2}$ we can write $F(z)=\sum_{l, k \geq 0} a_{l, k} z_{1}^{l} z_{2}^{k}$ in a small neighbourhood around 0. Using (4.1) we find that $a_{l, k}=0$ for $m \neq l+n k$, i.e. only finite many $a_{l, k}$ 's are different from 0 . From the identity theorem for holomorphic functions we find $F=\left.\hat{F}\right|_{\overline{B^{2}}}$ with $\hat{F} \in \mathbb{C}_{m}\left[z_{1}, z_{2}\right]$ and $\ell^{*} \hat{F}=$ $F_{\left.\right|_{X}}=f$. 
From now on we assume $n \geq 1$. Since $\mu$ is transversal we find that a global frame for $\mathbb{C} T X$ is given by $(Z, \bar{Z}, T)$, where $Z$ (resp. $\bar{Z}$ ) is a frame for $T^{1,0} X$ (resp. $\left.T^{0,1} X\right)$. We want to construct an $S^{1}$-invariant Hermitian metric $\langle\cdot \mid \cdot\rangle$ on $\mathbb{C} T X$ such that

(*) $T^{1,0} X \perp T^{0,1} X, T \perp\left(T^{1,0} X \oplus T^{0,1} X\right),\langle T \mid T\rangle=1$,

$(* *)\langle u \mid v\rangle$ is real if $u, v$ are real tangent vectors.

We do so by defining $(Z, \bar{Z}, T)$ to be an orthonormal frame. Then, (*) and $(* *)$ are satisfied. Moreover, the assumptions on $\gamma$ and the construction of $Z$ imply

$$
d \mu\left(e^{i \theta}, \cdot\right)_{z} Z_{z}=\lambda\left(e^{i \theta}, z\right) Z_{\mu\left(e^{i \theta}, z\right)}
$$

for some smooth function $\lambda$ on $S^{1} \times X$ with $|\lambda| \equiv 1$. Thus, the metric is rigid. Note that for the $S^{1}$-actions considered in this example, any rigid Hermitian metric which satisfies $(*)$ and $(* *)$ can be obtained in this way.

For $z \neq 0$ we define

$$
\alpha_{z}=\frac{\gamma(z)}{\left|z_{1}\right|^{2}+n\left|z_{2}\right|^{2}}\left(n z_{2} d z_{1}-z_{1} d z_{2}\right) \in T_{z}^{1,0^{*}} \mathbb{C}^{2}
$$

and

$$
\tilde{\omega}_{z}=-\frac{i}{2\left(\left|z_{1}\right|^{2}+n\left|z_{2}\right|^{2}\right)}\left(z_{1} d \bar{z}_{1}-\bar{z}_{1} d z_{1}+z_{2} d \bar{z}_{2}-\bar{z}_{2} d z_{2}\right) \text {. }
$$

Furthermore, we set $Z^{*}=\ell^{*} \alpha, \bar{Z}^{*}=\ell^{*} \bar{\alpha}$ and $\omega_{0}=\ell^{*} \tilde{\omega}$.

Lemma 4.6. $\left(Z^{*}, \bar{Z}^{*},-\omega_{0}\right)$ is the dual frame for $(Z, \bar{Z}, T)$.

Proof. A direct calculation shows $\omega_{0}(Z)=\omega_{0}(\bar{Z})=0, \omega_{0}(T)=-1$, $Z^{*}(T)=\bar{Z}^{*}(T)=0, Z^{*}(\bar{Z})=\bar{Z}^{*}(Z)=0$ and $Z^{*}(Z)=\bar{Z}^{*}(\bar{Z})=1$.

Using this lemma we can compute the Levi form $\mathcal{L}$ and its determinant:

Lemma 4.7. One has

$$
\left|\operatorname{det} \mathcal{L}_{z}\right|=\frac{1}{2} \frac{|\gamma(z)|^{-2}}{\left|z_{1}\right|^{2}+n\left|z_{2}\right|^{2}} .
$$

Proof. Consider

$$
\mathcal{L}_{z}=\left.\frac{i}{2} d \omega_{0}\right|_{T_{z}^{1,0} X \times T_{z}^{0,1} X}
$$




$$
\begin{aligned}
& =\left.\frac{1}{2\left(\left|z_{1}\right|^{2}+n\left|z_{2}\right|^{2}\right)}\left(d z_{1} \wedge d \bar{z}_{1}+d z_{2} \wedge d \bar{z}_{2}\right)\right|_{T_{z}^{1,0} X \times T_{z}^{0,1} X} \\
& =\frac{1}{2} \frac{|\gamma(z)|^{-2}}{\left|z_{1}\right|^{2}+n\left|z_{2}\right|^{2}} Z_{z}^{*} \wedge \bar{Z}_{z}^{*} .
\end{aligned}
$$

We define an orientation on $X$ by saying $(Z, \bar{Z}, T)$ is an oriented frame. Then the volume form of $X$ is given by $d V_{X}=-\frac{i}{2}\left(Z^{*}+\bar{Z}^{*}\right) \wedge\left(Z^{*}-\bar{Z}^{*}\right) \wedge\left(-\omega_{0}\right)=-i Z^{*} \wedge \bar{Z}^{*} \wedge \omega_{0}=-i \ell^{*}(\alpha \wedge \bar{\alpha} \wedge \tilde{\omega})$.

In the next section we need to compute several integrals on $X$. Thus, it is useful to have the following expression,

Lemma 4.8. One has

$$
\begin{aligned}
(\alpha \wedge \bar{\alpha} \wedge \tilde{\omega})_{z}=-\frac{i}{2}\left(\frac{|\gamma(z)|}{\left|z_{1}\right|^{2}+n\left|z_{2}\right|^{2}}\right)^{2} & \left(\left(z_{1} d \bar{z}_{1}-\bar{z}_{1} d z_{1}\right) \wedge d z_{2} \wedge d \bar{z}_{2}\right. \\
& \left.+n d z_{1} \wedge d \bar{z}_{1} \wedge\left(z_{2} d \bar{z}_{2}-\bar{z}_{2} d z_{2}\right)\right) .
\end{aligned}
$$

Proof. One calculates

$$
\begin{aligned}
& \frac{2 i\left(\left|z_{1}\right|^{2}+n\left|z_{2}\right|^{2}\right)^{3}}{|\gamma(z)|^{2}}(\alpha \wedge \bar{\alpha} \wedge \tilde{\omega})_{z} \\
& =\left(n^{2}\left|z_{2}\right|^{2} d z_{1} \wedge d \bar{z}_{1}+\left|z_{1}\right|^{2} d z_{2} \wedge d \bar{z}_{2}-n z_{2} \bar{z}_{1} d z_{1} \wedge \bar{z}_{2}-n z_{1} \bar{z}_{2} d z_{2} \wedge \bar{z}_{1}\right) \\
& \quad \wedge\left(z_{1} d \bar{z}_{1}-\bar{z}_{1} d z_{1}+z_{2} d \bar{z}_{2}-\bar{z}_{2} d z_{2}\right) \\
& =z_{2} n^{2}\left|z_{2}\right|^{2} d z_{1} \wedge d \bar{z}_{1} \wedge d \bar{z}_{2}-\bar{z}_{2} n^{2}\left|z_{2}\right|^{2} d z_{1} \wedge d \bar{z}_{1} \wedge d z_{2}+z_{1}\left|z_{1}\right|^{2} d \bar{z}_{1} \wedge d z_{2} \wedge d \bar{z}_{2} \\
& \quad-\bar{z}_{1}\left|z_{1}\right|^{2} d z_{1} \wedge d z_{2} \wedge d \bar{z}_{2}+n z_{2}\left|z_{1}\right|^{2} d z_{1} \wedge d \bar{z}_{1} \wedge d z_{2}-n \bar{z}_{1}\left|z_{2}\right|^{2} d z_{1} \wedge d z_{2} \wedge d \bar{z}_{2} \\
& \quad-n \bar{z}_{2}\left|z_{1}\right|^{2} d z_{1} \wedge d \bar{z}_{1} \wedge d z_{2}+n z_{1}\left|z_{2}\right|^{2} d \bar{z}_{1} \wedge d z_{2} \wedge d \bar{z}_{2} \\
& =\left(\left|z_{1}\right|^{2}+n\left|z_{2}\right|^{2}\right)\left(z_{1} d \bar{z}_{1} \wedge d z_{2} \wedge d \bar{z}_{2}-\bar{z}_{1} d z_{1} \wedge d z_{2} \wedge d \bar{z}_{2}\right. \\
& \left.\quad+n z_{2} d z_{1} \wedge d \bar{z}_{1} \wedge d \bar{z}_{2}-n \bar{z}_{2} d z_{1} \wedge d \bar{z}_{1} \wedge d z_{2}\right)
\end{aligned}
$$

\subsection{Computation of the Szegő kernel}

Recall that we assume $n \geq 1$. In this section we will construct an orthonormal basis for $H_{b, m}^{0}(X)$. Therefore we choose $\gamma \in C^{\infty}\left(\mathbb{C}^{2}\right)$ (see Section 
4.1) such that

$$
\gamma(z)=\sqrt{\left|z_{1}\right|^{2}+n\left|z_{2}\right|^{2}}
$$

on $X$. Then all the assumptions on $\gamma$ stated in Section 4.1 are satisfied.

Fix $m \geq 0$. For $0 \leq k \leq\left\lfloor\frac{m}{n}\right\rfloor$ define $s_{k} \in H_{b, m}^{0}(X)$ by

$$
s_{k}(z)=\sqrt{a_{k}} z_{1}^{m-n k} z_{2}^{k}, a_{k}=\frac{m+(1-n) k+1}{4 \pi^{2}}\left(\begin{array}{c}
m+(1-n) k \\
k
\end{array}\right) .
$$

One has the following lemma which we will prove in the end of this section.

Lemma 4.9. The set $\left\{s_{0}, s_{1}, \ldots, s_{\left\lfloor\frac{m}{n}\right\rfloor}\right\}$ is an orthonormal basis for $H_{b, m}^{0}(X)$.

Using this lemma we can write down the Szegö kernel (see Section 2.2).

Theorem 4.10. Fix $n \in \mathbb{N}, n \geq 1$. For the metric on $X$ constructed in Section 4.1 with $\gamma$ chosen as above and any $m \geq 0$ the Szegö kernel $S_{m} \in C^{\infty}(X \times X)$ for $H_{b, m}^{0}(X)$ is given by

$S_{m}(z, w)=\frac{1}{4 \pi^{2}} \sum_{k=0}^{\left\lfloor\frac{m}{n}\right\rfloor}\left(\begin{array}{c}m+(1-n) k \\ k\end{array}\right)(m+(1-n) k+1)\left(z_{1} \bar{w}_{1}\right)^{m-n k}\left(z_{2} \bar{w}_{2}\right)^{k}$

In the following we will prove Lemma 4.9.

Proof of Lemma 4.9. Consider the map

$$
\begin{aligned}
\psi:(0,1) \times(0,2 \pi)^{2} & \rightarrow X \\
(r, s, t) & \mapsto\left(r e^{i s}, \sqrt{1-r^{2}} e^{i t}\right) .
\end{aligned}
$$

Then for any $f \in C^{\infty}(X)$ one has

$$
\int_{X} f d V_{X}=\int_{0}^{1} \int_{0}^{2 \pi} \int_{0}^{2 \pi} \psi^{*}\left(f d V_{X}\right)
$$

i.e. we have to compute $\psi^{*}\left(d V_{X}\right)$. We write down

$$
\psi^{*} d z_{1}=e^{i s}(d r+i r d s), \psi^{*} d z_{2}=e^{i t}\left(-\frac{r}{\sqrt{1-r^{2}}} d r+i \sqrt{1-r^{2}} d t\right) .
$$

Thus,

$$
\psi^{*}\left(d z_{1} \wedge d \bar{z}_{1}\right)=-2 i r d r \wedge d s, \psi^{*}\left(d z_{2} \wedge d \bar{z}_{2}\right)=2 i r d r \wedge d t
$$


and

$$
\psi^{*}\left(z_{1} d \bar{z}_{1}-\bar{z}_{1} d z_{1}\right)=-2 i r^{2} d s, \psi^{*}\left(z_{2} d \bar{z}_{2}-\bar{z}_{2} d z_{2}\right)=-2 i\left(1-r^{2}\right) d t
$$

Using this we get

$$
\begin{aligned}
\psi^{*}\left(\left(z_{1} d \bar{z}_{1}-\bar{z}_{1} d z_{1}\right) \wedge d z_{2} \wedge d \bar{z}_{2}\right) & =-4 r^{3} d r \wedge d s \wedge d t \\
\psi^{*}\left(n d z_{1} \wedge d \bar{z}_{1} \wedge\left(z_{2} d \bar{z}_{2}-\bar{z}_{2} d z_{2}\right)\right) & =4 n\left(-r+r^{3}\right) d r \wedge d s \wedge d t
\end{aligned}
$$

which leads to

$$
\begin{aligned}
\psi^{*}\left(d V_{X}\right) & =-\frac{1}{2}\left(\frac{\left|\gamma\left(r e^{i s}, \sqrt{1-r^{2}} e^{i t}\right)\right|}{r^{2}+n\left(1-r^{2}\right)}\right)^{2}\left(-4 r^{3}+4 n r^{3}-4 n r\right) d r \wedge d s \wedge d t \\
& =2 r \frac{\left|\gamma\left(r e^{i s}, \sqrt{1-r^{2}} e^{i t}\right)\right|^{2}}{r^{2}+n\left(1-r^{2}\right)} d r \wedge d s \wedge d t \\
& =2 r d r \wedge d s \wedge d t
\end{aligned}
$$

where for the last line we used that $(\gamma \circ \psi)(r, s, t)=\sqrt{r^{2}+n\left(1-r^{2}\right)}$. Now we compute

$$
\begin{aligned}
\int_{X} s_{k} \overline{s l} d V_{X} & =\sqrt{a_{k} a_{l}} \int_{0}^{1} \int_{0}^{2 \pi} \int_{0}^{2 \pi} r^{2 m-n(k+l)}{\sqrt{1-r^{2}}}^{k+l} e^{i n(l-k) s} e^{i(k-l) t} 2 r d r d s d t \\
& = \begin{cases}0, & \text { for } k \neq l, \\
4 \pi^{2} a_{k} \int_{0}^{1}\left(r^{2}\right)^{m-n k}\left(1-r^{2}\right)^{k} 2 r d r, & \text { for } k=l .\end{cases}
\end{aligned}
$$

This shows directly that the $s_{k}$ are pairwise orthogonal. In order to prove $\left\|s_{k}\right\|=1,0 \leq k \leq\lfloor m / n\rfloor$ we set

$$
I(k, m-n k):=\int_{0}^{1}\left(r^{2}\right)^{m-n k}\left(1-r^{2}\right)^{k} 2 r d r
$$

for $0 \leq k \leq\lfloor m / n\rfloor$ and observe for $k>0$

$$
\begin{aligned}
I(k, m-n k) & =\int_{0}^{1} r^{m-n k}(1-r)^{k} d r \\
& =\left[\frac{r^{m-n k+1}(1-r)^{k}}{m-n k+1}\right]_{0}^{1}+\frac{k}{m-n k+1} \int_{0}^{1} r^{m-n k+1}(1-r)^{k-1} d r \\
& =\frac{k}{m-n k+1} I(k-1, m-n k+1)
\end{aligned}
$$


and $I(0, m-n k+k)=(m-n k+k+1)^{-1}$. By induction one gets

$$
I(k, m-n k)=\left(\left(\begin{array}{c}
m-n k+k \\
k
\end{array}\right)(m-n k+k+1)\right)^{-1}=\left(4 \pi^{2} a_{k}\right)^{-1}
$$

which finishes the proof of Lemma 4.9.

\subsection{Discussion of the general theory in context of the example}

For $n \geq 1$ we have that the CR $S^{1}$-action $\mu$ on the compact CR manifold $X=S^{3}$ is transversal (see Theorem 4.1). We also constructed a rigid Hermitian metric such that $T^{1,0} X \perp T^{0,1} X, T \perp\left(T^{1,0} X \oplus T^{0,1} X\right),\langle T \mid T\rangle=1$ and $\langle u \mid v\rangle$ is real if $u, v$ are real tangent vectors in Section 4.1. Theorem 4.10 provides an expression for the Szegö kernel:

$S_{m}(z, w)=\frac{1}{4 \pi^{2}} \sum_{k=0}^{\left\lfloor\frac{m}{n}\right\rfloor}\left(\begin{array}{c}m+(1-n) k \\ k\end{array}\right)(m+(1-n) k+1)\left(z_{1} \bar{w}_{1}\right)^{m-n k}\left(z_{2} \bar{w}_{2}\right)^{k}$.

From Lemma 4.7 and its proof we find that the CR structure is strictly pseudoconvex and that the determinant of the Levi form is given by

$$
\left|\operatorname{det} \mathcal{L}_{z}\right|=\frac{1}{2} \frac{1}{\left(\left|z_{1}\right|^{2}+n\left|z_{2}\right|^{2}\right)^{2}} .
$$

On the one hand, all the assumptions for applying Theorem 2.10 or 2.11 are satisfied. On the other hand we have an explicit expression for the Szegö kernel. We will now study the expression in several cases and compare it to the results stated in Theorem 2.10 and Theorem 2.11 in that specific case.

In the case $n=1$ one has $X_{\text {reg }}=X$ and (4.3) simplifies to

$$
S_{m}(z, w)=\frac{1}{2} \cdot \frac{m+1}{2 \pi^{2}}\left(z_{1} \bar{w}_{1}+z_{2} \bar{w}_{2}\right)^{m} .
$$

Because of $\left|\operatorname{det} \mathcal{L}_{z}\right|=\frac{1}{2}$, one observes that

$$
S_{m}(z, z)=\frac{m+1}{2 \pi^{2}} \cdot \frac{1}{2}=\frac{1}{2 \pi}\left(\frac{1}{\pi}\left|\operatorname{det} \mathcal{L}_{z}\right| m^{1}+\frac{1}{\pi}\left|\operatorname{det} \mathcal{L}_{z}\right| m^{0}\right)
$$

which verifies Theorem 2.10 and shows that the leading term of the expansion 
of $S_{m}(z, z)$ coincides with the term stated in (2.16). Given $n \geq 2$ one considers the following two cases:

For $z \in X_{n}$ and $w \in X$ one has

$$
S_{m}(z, w)= \begin{cases}0, & \text { for } n \nmid m, \\ \left(\frac{m}{n}+1\right) \frac{\left(z_{2} \bar{w}_{2}\right)^{\frac{m}{n}}}{4 \pi^{2}}, & \text { else, }\end{cases}
$$

and $\left|\operatorname{det} \mathcal{L}_{z}\right|=1 /\left(2 n^{2}\right)$. Thus, for $z \notin X_{\text {reg }}$

$$
S_{m}(z, z)=\frac{m+n}{2 \pi^{2}} \frac{\chi_{m, n}}{2 n^{2}}=\frac{\chi_{m, n}}{2 \pi}\left(\frac{1}{\pi}\left|\operatorname{det} \mathcal{L}_{z}\right| m^{1}+\frac{n}{\pi}\left|\operatorname{det} \mathcal{L}_{z}\right| m^{0}\right)
$$

where $\chi_{m, n}=n$ for $n \mid m$ and $\chi_{m, n}=0$ otherwise, which coincides with the behaviour of the Szegö kernel expansion on $X \backslash X_{\text {reg }}$ predicted in Theorem 2.11 .

By way of comparison, for $z, w \in X$ with $\left|z_{1}\right|=1$ (which implies $z \in$ $X_{\text {reg }}$ ) one finds

$$
S_{m}(z, w)=\frac{m+1}{4 \pi^{2}}\left(z_{1} \overline{w_{1}}\right)^{m}
$$

and $\left|\operatorname{det} \mathcal{L}_{z}\right|=1 / 2$ which leads to

$$
S_{m}(z, z)=\frac{m+1}{2 \pi^{2}} \cdot \frac{1}{2}=\frac{1}{2 \pi}\left(\frac{1}{\pi}\left|\operatorname{det} \mathcal{L}_{z}\right| m^{1}+\frac{1}{\pi}\left|\operatorname{det} \mathcal{L}_{z}\right| m^{0}\right),
$$

i.e. $S_{m}(z, z)$ has an asymptotic expansion as described in Theorem 2.10.

Vice versa, one can apply Theorem 2.10 in order to deduce results on the asymptotic behavior of certain expressions in elementary analysis. Let $m, n \geq 1$ be integer and $a \in(0,1)$ a real number. Consider the expression

$$
A(m, n, a):=\sum_{k=0}^{\left\lfloor\frac{m}{n}\right\rfloor}\left(\begin{array}{c}
m+(1-n) k \\
k
\end{array}\right)(m+(1-n) k+1)(1-a)^{m-n k} a^{k} .
$$

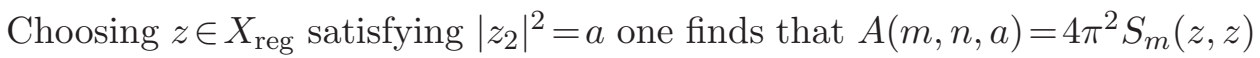
holds. Thus, using Theorem 2.10 we can study the behavior of $A(m, n, a)$ for $m \rightarrow \infty$. One has for example

$$
\lim _{m \rightarrow \infty} m^{-1} A(m, n, a)=(1+(n-1) a)^{-2} .
$$




\section{Acknowledgment}

Parts of this work were finished when the authors visited the Institute of Mathematics, Academia Sinica during the spring vacation 2016. The authors would like to express their gratitude to the institute for hospitality, a comfortable accommodation and financial support during their visit.

\section{References}

1. M. Adachi, On the ampleness of positive CR line bundles over Levi-flat manifolds, Publ. Res. Inst. Math. Sci., 50 (2014), no. 1, 153-167.

2. A. Andreotti and Y. -T. Siu, Projective embeddings of pseudoconcave spaces, Ann. Sc. Norm. Super. Pisa, 24 (1970), 231-278.

3. M.-S. Baouendi, L.-P. Rothschild and F. Treves, CR structures with group action and extendability of CR functions, Invent. Math., 83 (1985), 359-396.

4. R. Berman, Holomorphic Morse inequalities on Manifolds with boundary, Ann. Inst. Fourier (Grenbole), 55 (2005), no. 4, 1055-1103.

5. L. Boutet de Monvel, Intégration des équations de Cauchy-Riemann induites formelles, Séminaire Goulaouic-Lions-Schwartz 1974-1975; Équations aux derivées partielles linéaires et non linéaires, Centre Math., École Polytech., Paris (1975), no. 9.

6. L. Boutet de Monvel and J. Sjöstrand, Sur la singularité des noyaux de Bergman et de Szegö, Société Mathématique de France, Astérisque, 34-35 (1976), 123-164.

7. D. M. Burns, Global behavior of some tangential Cauchy-Riemann equations, Lecture Notes in Pure and Appl. Math., 48 (1979), 51-56.

8. S.-C. Chen and M.-C. Shaw, Partial differential equations in several complex variables, AMS/IP Studies in Advanced Mathematics, 19 (American Mathematical Society International Press, 2001).

9. J.-H. Cheng, C.-Y. Hsiao and I.-H. Tsai, Heat kernel asymptotics, local index theorem and trace integrals for Cauchy-Riemann manifolds with $S^{1}$ action, Société, Mathématique de France (2019), no. 162.

10. J.-P. Demailly, Champs magnétiques et inegalités de Morse pour la $d^{\prime \prime}$-cohomology, Ann. Inst. Fourier (Grenoble) 35 (1985), 189-229.

11. J.-P. Demailly, Holomorphic morse inequalities, Proc. Sympos. Pure Math., 52 (1991), 93-144.

12. S. Dragomir and G. Tomassini, Differential geometry and analysis on CR manifolds, Progress in Mathematics, 246 (Birkhäuser Basel - 2006).

13. J.-J. Duistermaat and G.-J. Heckman, On the variation in the cohomology of the symplectic form of thereduced phase space, Invent. Math., 69 (1982), 259-268. 
14. K. Fritsch and P. Heinzner, Equivariant embeddings of strongly pseudoconvex CauchyRiemann manifolds, arXiv:2002.00219.

15. K. Fritsch, H. Herrmann and C.-Y. Hsiao, G-equivariant embedding theorems for CR manifolds of high codimension, arXiv:1810.09629.

16. H. Grauert, Über Modifikationen und exzeptionelle analytische Mengen, Math. Ann., 146 (1962), 331-368.

17. E. Getzler, An analoque of Demailly's inequality for strictly pseudoconvex CR manifolds, J. Differential Geom., 29 (1989), No. 2, 231-244.

18. C.-Y. Hsiao, Szegő kernel asymptotics for high power of CR line bundles and Kodaira embedding theorems on CR manifolds, Mem. Amer. Math. Soc., 254 (2018), no. 1217.

19. C.-Y. Hsiao and G. Marinescu, Szegö kernel asymptotics and Morse inequalties on CR manifolds, Math. Z., 271 (2012), 509-553.

20. H. Herrmann, C.-Y. Hsiao and X. Li, Szegő kernel asymptotic expansion on strongly pseudoconvex CR manifolds with $S^{1}$ action, Internat. J. Math., 29 (2018), no. 9, 1850061.

21. H. Herrmann, C.-Y. Hsiao and X. Li, Szegö kernel expansion and equivariant embedding of CR manifolds with circle action, Ann. Global Anal. Geom., 52 (2017), no. 3, 313-340.

22. H. Herrmann, C.-Y. Hsiao and X. Li, Szegő kernels and equivariant embedding theorems for CR manifolds, arXiv:1710.04910.

23. C.-Y. Hsiao and X. Li, Szegö kernel asymptotics and Morse inequalities on CR manifolds with $S^{1}$ action, Asian J. Math., 22 (2018), no. 3, 413-450.

24. C.-Y. Hsiao and X. Li, Morse inequalities for Fourier components of Kohn-Rossi cohomology of CR manifolds with $S^{1}$-action, Math. Z., 284 (2016), no. 1-2, 441-468.

25. C.-Y. Hsiao, X. Li and G. Marinescu, Equivariant Kodaira embedding of CR manifolds with circle action, to appear in Michigan Mathematical Journal, arXiv:1603.08872, doi: $10.1307 / \mathrm{mmj} / 1587628815$.

26. C.-Y. Hsiao, R.-T. Huang, X. Li and G. Shao, $S^{1}$-equivariant Index theorems and Morse inequalities on complex manifolds with boundary, arXiv:1711.05537. J. Funct. Anal., 279 (2020), no. 3, 108558, 51 pp.

27. C. D. Hill and M. Nacinovich, A weak pseudoconcavity condition for abstract almost CR manifolds, Invent. Math., 142 (2000), 251-283.

28. J. J. Kohn, The range of Cauchy-Riemann operator, Duke Math. J., 53 (1986), no. 2, 525-545.

29. J. J. Kohn, Estimates for $\bar{\partial}_{b}$ on pseudoconvex CR manifolds. Pseudodifferential operators and applications, Proc. Sympos. Pure Math., 43 (Amer. Math. Soc., Providence, RI, 1985), 207-217.

30. L. Lempert, On three dimensional Cauchy-Riemann manifolds, Jour. Amer. Math. Soc., 5 (1992), no. 4, 923-969. 
31. G. Marinescu, Asymptotics Morse inequalities for pseudoconcave manifolds, Ann Scuola Norm. Sup. Pisa C1. Sci., 23 (1996), no. 1, 27-55.

32. X. Ma and G. Marinescu, Holomorphic Morse inequalities and Bergman kernels, Progress in Mathematics, 254 (Birkhäuser Verlag, Basel, 2007).

33. A. Melin and J. Sjöstrand, Fourier integral operators with complex-valued phase functions, Springer Lecture Notes in Math., 459 (1975), 120-223.

34. J. Morrow and K. Kodaira, Complex Manifolds, AMS Chelsea Publishing Series 355 (American Mathematical Soc., 2006).

35. T. Ohsawa and N. Sibony, Kähler identity on Levi flat manifolds and application to the embedding, Nagoya Math. J., 158 (2000), 87-93.

36. H. Rossi, Attaching analytic spaces to an analytic space along a pseudoconvex boundary, Proc. Conf. on Complex Manifolds (Springer Berlin, Heidelberg, 1965), 242-256.

37. Y.-T. Siu, A vanishing theorem for semipositive line bundles over non-Kähler manifolds, J. Differential Geom., 19 (1984), no. 2, 431-452.

38. Z. Wang and X. Zhou, CR eigenvalue estimate and Kohn-Rossi cohomology, to appear in J. Differential. Geom., arXiv:1905.03474. 